\title{
On automated analysis of flow patterns in cerebral aneurysms based on vortex identification
}

\author{
Gwen Mulder • Arjen C. B. Bogaerds • \\ Peter Rongen - Frans N. van de Vosse
}

Received: 7 April 2008 / Accepted: 27 January 2009 / Published online: 4 March 2009

(C) The Author(s) 2009. This article is published with open access at Springerlink.com

\begin{abstract}
It is hypothesized that the risk of rupture of cerebral aneurysms is related to geometrical and mechanical properties of the arterial wall as well as to local hemodynamics. In order to gain better understanding of the hemodynamical factors involved in intra-aneurysmal flows, a thorough analysis of the 3D velocity field within an idealized geometry is needed. This includes the identification and quantification of features like vortices and stagnation regions. The aim of our research is to develop experimentally validated computational methods to analyse intra-aneurysmal vortex patterns and, eventually, define candidate hemodynamical parameters (e.g. vortex strength) that could be predictive for rupture risk. A computational model based on a standard Galerkin finite-element approximation and an Euler implicit time integration has been applied to compute the velocity field in an idealized aneurysm geometry and the results have been compared to Particle Image Velocimetry (PIV) measurements in an in vitro model. In order to analyze the vortices observed in the aneurysmal sac, the vortex identification scheme as proposed by Jeong and Hussain (JFM 285:69-94, 1995) is applied. The 3D intra-aneurysmal velocity fields reveal complex vortical structures. This study indicates that the computational method predicts well the vortex structure that is found in the in vitro model and that a $3 \mathrm{D}$ analysis method like the vortex identification as proposed is needed to fully understand and quantify the vortex dynamics of intra-aneurysmal flow. Furthermore, such an automated analysis method would allow the definition of parameters predictive for rupture in clinical practice.
\end{abstract}

Keywords Cerebral aneurysm $\cdot$ Computational fluid dynamics $\cdot$ Particle image velocimetry $\cdot$ Vortex identification

\section{Introduction}

Cerebral aneurysms are localized pathological dilatations of cerebral arteries, most commonly found in the circle of Willis. In the general population, approximately $2-5 \%$ is likely to harbor these aneurysms $[1,2]$, which have an annual rupture risk of approximately 1\% [3]. Rupture of a cerebral aneurysm results in subarachnoid hemorrhage $(\mathrm{SAH})$, with a mortality rate of $40-50 \%[4,5]$.

G. Mulder $(\varangle)$. A. C. B. Bogaerds · F. N. van de Vosse

Department of Biomedical Engineering, Eindhoven University of Technology, Eindhoven, The Netherlands

e-mail: g.mulder@tue.nl

P. Rongen

Philips Medical Systems, Best, The Netherlands 


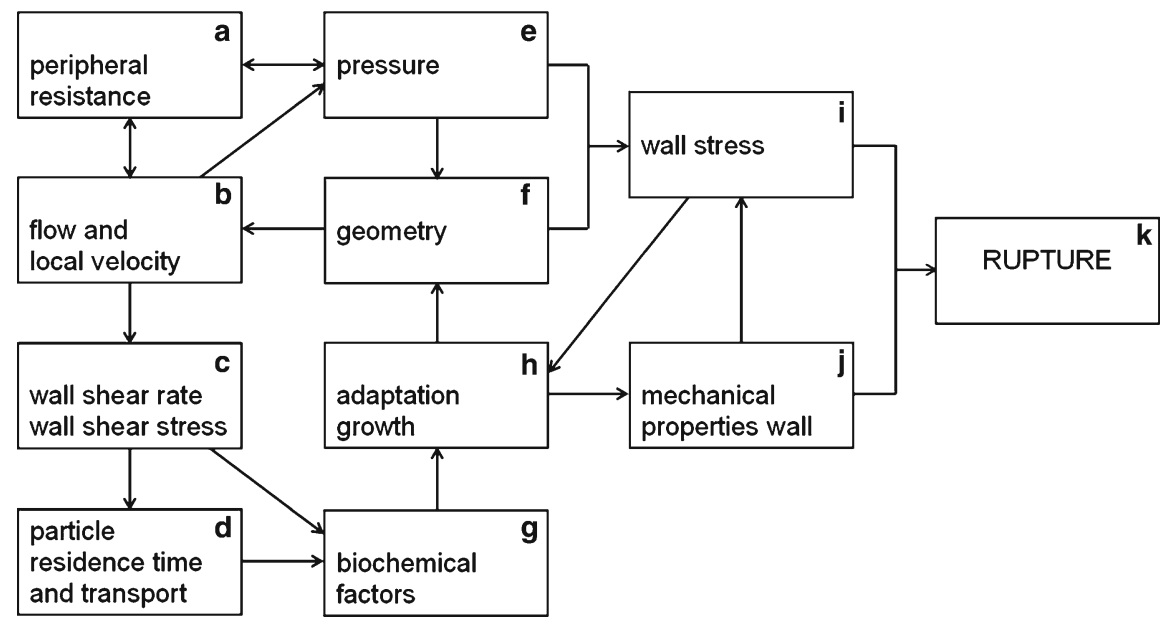

Fig. 1 Schematic representation of the factors involved in aneurysm rupture

The rupture risk is determined by the loading state and the mechanical properties of the arterial wall, which are both related to the hemodynamics as illustrated in Fig. 1. The loading state, i.e., wall stress $(i)$, depends on the mechanical properties of the arterial wall $(j)$, the aneurysmal geometry $(f)$ and the intra-aneurysmal pressure $(e)$. The pressure is determined by the flow $(b)$ through the parent artery and the peripheral resistance $(a)$. The influence of the peripheral circulation is not considered in most Computational Fluid Dynamics (CFD) models since they focus on an isolated rigid aneurysm geometry [6,7]. Coupling the 3D model to a 1D model of the global cerebral circulation would allow prescribing more realistic boundary conditions. Furthermore, autoregulation controlling the resistance provides feedback of the pressure and flow. The local geometry, which slightly varies with the pressure due to the distensibility of the arterial wall, has a major effect on the intra-aneurysmal flow patterns $(b)$ [8]. However, this geometry is also affected by the flow via biochemical cascades that control the adaptation of the arterial wall (mechano-transduction) $[9,10]$. The flow-induced wall shear rate $(c)$ is known to affect particle residence times $(d)$. Moreover, changes in shear-stress $(c)$ magnitude and direction alter the permeability of the arterial wall and the transport $(d)$ between the lumen and wall [11]. Endothelial cells are sensitive to these hemodynamical changes, resulting in the activation of biochemical factors $(h)$ that control the adaptation of the arterial wall $(g)$. Altogether, this adaptation may become pathological and may cause weakening of the arterial wall, which, under the influence of wall stress, may result in aneurysm growth. In the event of rupture $(k)$, the mechanical properties of the arterial wall have been altered by the degradation process such that the stress in the wall exceeds its strength.

One would prefer to base the decision whether or not to treat an aneurysm on the balance between the riskof-rupture and the risk related to the treatment itself. However, the risk of rupture is not easily determined, since there currently are no proven methods for in vivo measurements of flow, pressure or mechanical properties of the wall in cerebral aneurysms. Several attempts have been made to find a direct correlation between geometry and risk of rupture, using parameters like size of the dome and aspect ratio [12-14]. However, no conclusive critical size parameter could be defined based on those studies. Currently, the decision whether treatment is recommended is based primarily on the size of an aneurysm, although this remains controversial.

Since rupture risk is also related to hemodynamical factors, intra-aneurysmal velocity fields in idealized as well as patient-specific models have been analyzed in various studies [15-17]. Since several methods for in vivo flow measurements in cerebral arteries are in development, it seems realistic to use these flow measurements as input for numerical models of which the geometry has been determined by e.g. CT imaging or 3D Rotational Angiography (3D-RA). In general, the intra-aneurysmal velocity fields show complex 3D flow patterns containing inflow jets, vortices, and stagnation regions. A quantitative comparison of 3D-RA images recorded with a high frame rate and numerical results shows similar flow patterns, which suggests that these major features are captured by the CFD models [18]. Cebral et al. [6] suggested a direct relation between intra-aneurysmal flow patterns and rupture risk. 
The flow patterns in the aneurysmal sac of patient-specific geometries were characterized based on the stability of inflow jet and the number of vortices. Unstable flow patterns could be related to aneurysm progression and rupture due to elevated oscillating stresses or larger regions of elevated mean wall shear stresses. Stable patterns may provide a more suitable environment for arterial adaptation mechanisms to counterbalance the stresses, resulting in safer aneurysms. Indeed, simple stable flow patterns, large impingement regions and large jet sizes are more commonly found in unruptured aneurysms, whereas disturbed flow patterns, small impingement regions and narrow jets were found more frequently in ruptured aneurysms. However, further research, evaluating more patientspecific geometries, is needed to confirm these preliminary results. In order to enable a more thorough and efficient analysis of the intra-aneurysmal velocity fields, these features should be identified in an automated fashion. Vortex and stagnation region identification allow a more accurate analysis of patient data, and therefore, are believed to enhance our understanding of the flow patterns observed in various geometries and sites at which rupture occurs most frequently.

In this research, a vortex identification scheme as proposed by Jeong and Hussain [19] has been implemented in order to evaluate the intra-aneurysmal velocity field in an idealized CFD model of a lateral aneurysm with a curved parent artery. The velocity field computed with this model is compared to the velocity field measured with Particle Image Velocimetry (PIV) in order to validate the observed vortex structure and check for possibly missed transitional flow features. Obviously, the geometry of both the parent artery and aneurysm have a high impact on the flow patterns, resulting in e.g. underestimation of the wall shear stress and complexity of the flow pattern in idealized geometries [20,21]. However, the rigid-walled idealized model is more suitable for examining the value of such an identification scheme in the analysis of aneurysmal flow patterns. Furthermore, this paper argues the commonly employed method of reviewing a single cross-section in the analysis of the complex 3D intra-aneurysmal flow.

Eventually, this research should lead to a more accurate method to estimate the risk of rupture of cerebral aneurysms. When accurate in vivo measurement of blood flow in the, frequently small, parent arteries becomes possible, the shear rate experienced by the endothelial cells covering the aneurysmal wall can be derived. The response of those endothelial cells leads to adaptation, or, in aneurysms, degradation of the arterial wall. Hence, patient-specific CFD modeling will become a valuable tool in risk-of-rupture assessment when models describing the relation of this degradation process to the hemodynamics become available. In clinical practice, however, the flow analysis should be based on automated methods like the vortex identification method presented in this study.

\section{Materials and methods}

\section{$2.1 \mathrm{CFD}$}

In general, the flow characteristics in lateral aneurysms depend on the geometrical configuration of the aneurysm in relation to the parent vessel, the size of the neck, the volume of the aneurysm and the hemorheological properties. The geometry used here (Fig. 2) was based on the geometrical considerations described by Parlea et al. [22].

The intra-aneurysmal velocity field is obtained by solving the momentum equation

$\rho\left\{\frac{\partial \boldsymbol{v}}{\partial t}+\boldsymbol{v} \cdot \nabla \boldsymbol{v}\right\}=-\nabla p+\nabla \cdot \boldsymbol{\tau}+\boldsymbol{f}$

and the incompressibility constraint:

$\nabla \cdot \boldsymbol{v}=0$

on the 3-dimensional domain $\Omega\left(\Omega \subset \mathbb{R}^{3}\right.$ constrained by the closed boundary $\Gamma$ ). Here, $\rho$ denotes the constant density, $\boldsymbol{v}$ the velocity vector, $\boldsymbol{f}$ a body force defined per unit of volume, $p$ the hydrostatic pressure and $\tau$ the extra stress tensor. Since Newtonian fluid behavior is assumed, the relation for the extra stress tensor reads: 

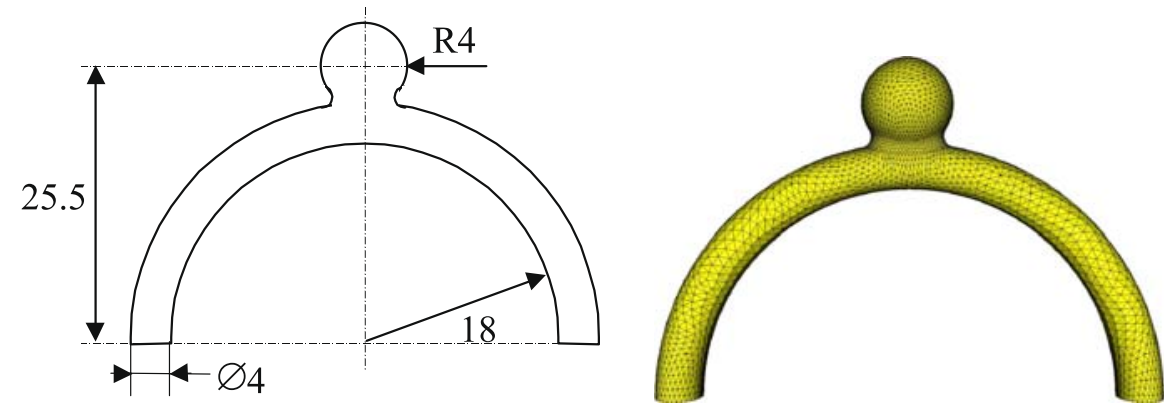

Fig. 2 Geometry of the lateral aneurysm model in [mm]. Measured from the neck, the aneurysm height and semi-axis height equal 7 and $3 \mathrm{~mm}$ respectively, while the neck width is $3.9 \mathrm{~mm}$. The 3D finite-element mesh generated with Patran (MSC Software) consists of 39,405 tetrahedron elements, with a total of 152,095 degrees of freedom

$\boldsymbol{\tau}=2 \eta \boldsymbol{D}$,

with $\eta$ the dynamic viscosity and $\boldsymbol{D}=\left(\nabla \boldsymbol{v}+\nabla \boldsymbol{v}^{T}\right) / 2$ the rate of deformation tensor. Substitution of (3) in (1) while neglecting body forces like gravity results in the well-known incompressible Navier-Stokes equations. Together with the appropriate boundary conditions on $\Gamma$ and suitable initial conditions, the Navier-Stokes equations will result in a unique solution for $\boldsymbol{v}$ and $p$ when the Reynolds numbers are sufficiently low. The weak formulation of the Navier-Stokes equations is found after definition of the appropriate Sobolev space of functions with $1^{\text {st }}$ order square-integrable derivatives on $\Omega\left(\boldsymbol{W}=\left\{\boldsymbol{w} \in\left[H_{0}^{1}(\Omega)\right]^{3}\right\}\right)$ that vanish on $\Gamma$ where Dirichlet boundary conditions are prescribed, as well as $Q$, the Lebesgue space of square-integrable functions on $\Omega\left(Q=\left\{q \in L^{2}(\Omega), \int_{\Omega} q \mathrm{~d} \Omega=0\right\}\right)$. In that case, the weak form is given by

$$
\begin{aligned}
& \int_{\Omega} \rho \boldsymbol{w} \cdot\left\{\frac{\partial \boldsymbol{v}}{\partial t}+\boldsymbol{v} \cdot \nabla \boldsymbol{v}\right\} \mathrm{d} \Omega+\int_{\Omega} \eta \nabla \boldsymbol{w}^{T}: \nabla \boldsymbol{v} \mathrm{d} \Omega-\int_{\Omega} p \nabla \cdot \boldsymbol{w} \mathrm{d} \Omega \\
& =\int_{\Gamma} \boldsymbol{w} \cdot(-p \boldsymbol{n}+\boldsymbol{\tau} \cdot \boldsymbol{n}) \mathrm{d} \Gamma \quad \forall \boldsymbol{w} \in \boldsymbol{W}, \\
& \int_{\Omega} q \nabla \cdot \boldsymbol{v} \mathrm{d} \Omega=0 \quad \forall q \in Q .
\end{aligned}
$$

The variational form is solved using Crouzeix-Raviart-type finite elements. These elements apply second-order continuous interpolation for the fluid velocity and a linear discontinuous basis for pressure interpolation. In this work, the domain $\Omega$ is discretized using tetrahedral elements with 15 nodal points. The 3 -dimensional computational grid is shown in Fig. 2.

At the arterial wall and the wall of the aneurysm, no-slip boundary conditions were applied. At the proximal side of the parent artery a sinusoidal start-up Poiseuille profile was prescribed, reaching a steady state limit after $1 \mathrm{~s}$. At the distal side of the parent artery, the normal component of the stress vector, and all in-plane velocity components, were prescribed as equal to zero. Based on the data reported by [23-25], the mean blood flow through the $4 \mathrm{~mm}$ parent artery was chosen to be $3.6 \mathrm{ml} \mathrm{s}^{-1}$. The radius of the parent artery, the mean blood velocity, and the blood viscosity (3.5 mPas) result in a Reynolds number $(\operatorname{Re}=\rho V R / \eta)$ of $\operatorname{Re}=165$.

Temporal discretization of the Navier-Stokes equations was achieved using the implicit Euler scheme, while Newton's method was used for the linearization of the convective terms within each time step. The iterative method used to solve the linearized set of equations is Bi-CGStab with an incomplete LU decomposition pre-conditioner [26]. 


\subsection{Vortex identification}

The vortex identification method developed by Jeong and Hussain [19] is based on the second largest eigenvalue of $\boldsymbol{D}^{2}+\boldsymbol{\Omega}^{2}$, with $\boldsymbol{D}$ and $\boldsymbol{\Omega}=\left(\nabla \boldsymbol{v}-\nabla \boldsymbol{v}^{T}\right) / 2$ the deformation and rotation tensor, respectively. After substitution of the vorticity equation, the gradient of the Navier-Stokes equations read

$\frac{\partial \boldsymbol{D}}{\partial t}+(\mathbf{v} \cdot \nabla) \boldsymbol{D}+\boldsymbol{D}^{2}+\boldsymbol{\Omega}^{2}=-\frac{1}{\rho} \nabla(\nabla p)+\frac{\eta}{\rho} \nabla \nabla^{2} \mathbf{v}$.

The Hessian of the pressure $H_{p}=\nabla(\nabla p)$ provides information about local pressure minima within the flow. In general, pressure gradients can be attributed to local irrotational straining (first two terms of the left hand side of Eq. 6), viscous dissipation (last term on the right-hand side) and rotational effects in the vortex cores. Hence, the rotational effects on the pressure gradient are represented in

$H_{p}^{\text {rot }}=-\rho\left(\boldsymbol{D}^{2}+\boldsymbol{\Omega}^{2}\right)$.

In order to find a local pressure minimum due to rotation around the vortex core two negative eigenvalues of $-H_{p}^{\text {rot }}$ are required. Hence,

$\lambda_{i}=\operatorname{eig}\left(\boldsymbol{D}^{2}+\boldsymbol{\Omega}^{2}\right), \quad$ with $\lambda_{2}<\mathbf{0} \quad\left(\lambda_{1}<\lambda_{2}<\lambda_{3}\right)$

results in a surface $\left(\lambda_{2}<0\right)$ that constrains the position of the vortex core(s).

\subsection{Experiment}

An in vitro model of an aneurysm on a curved parent artery (Fig. 2) was produced out of silicone (Sylgard 184). A stationary pump (Cole-Palmer, Mo.75211-15) was used to produce a stationary flow with $\mathrm{Re}=165$.

A $30 \mathrm{wt} \%$ electrolyte solution of calcium chloride and magnesium chloride (ratio 5:1) was selected as working fluid, minimizing the difference in refraction indices. In order to enable the PIV measurements, the fluid was seeded with silver-coated hollow glass particles with mean particle size $10 \mu \mathrm{m}$ and density $1.4 \times 10^{3} \mathrm{~kg} \mathrm{~m}^{-3}$ (DANTEC). The particles were illuminated by a continuous argon ion laser (Midwest ILT 5500A; 458-515 nm; 300 mJ/s) and recorded by a high-speed video camera (Phantom V9.0).

\subsection{Data acquisition and postprocessing}

The three measured planes correspond to plane b, e and $\mathrm{h}$ in Fig. 3. The high-speed video camera allows high frame rates providing a temporal resolution usually not achieved in PIV. Plane $\mathrm{b}$ was measured using a frame rate of $5.4 \mathrm{kHz}$, while $5 \mathrm{kHz}$ was used for the other planes. Depending on the expected velocities within the measurement
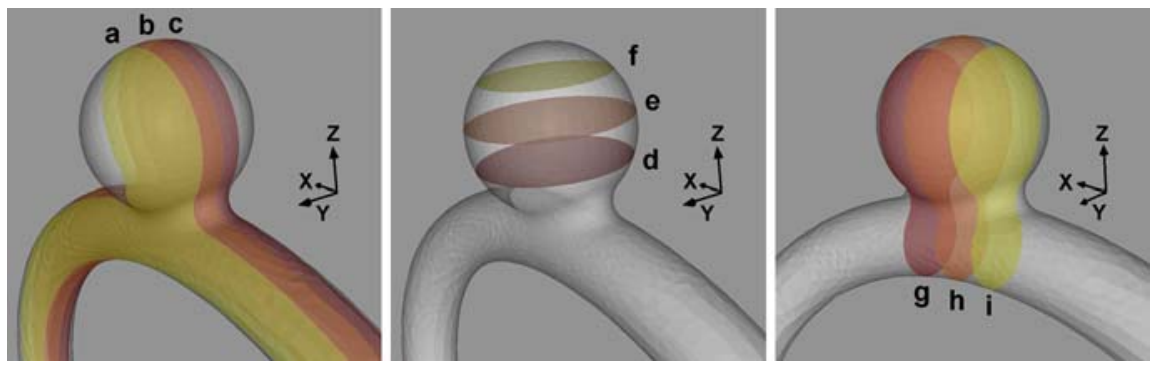

Fig. 3 The velocity fields are visualized on 9 cross-sections, of which the numbering corresponds to the numbering in Figs. 5 and 7 
Fig. 4 The absolute in-plane velocity (in $\mathrm{ms}^{-1}$ ) determined with CFD and PIV in planes $b$, e, and $h$, as defined in Fig. 3. The velocity vector scaling in planes $\mathrm{e}$ and $\mathrm{h}$ is a factor 3 higher for visualization purposes. The flow in the parent artery, as shown in the profiles (top), is from left to right
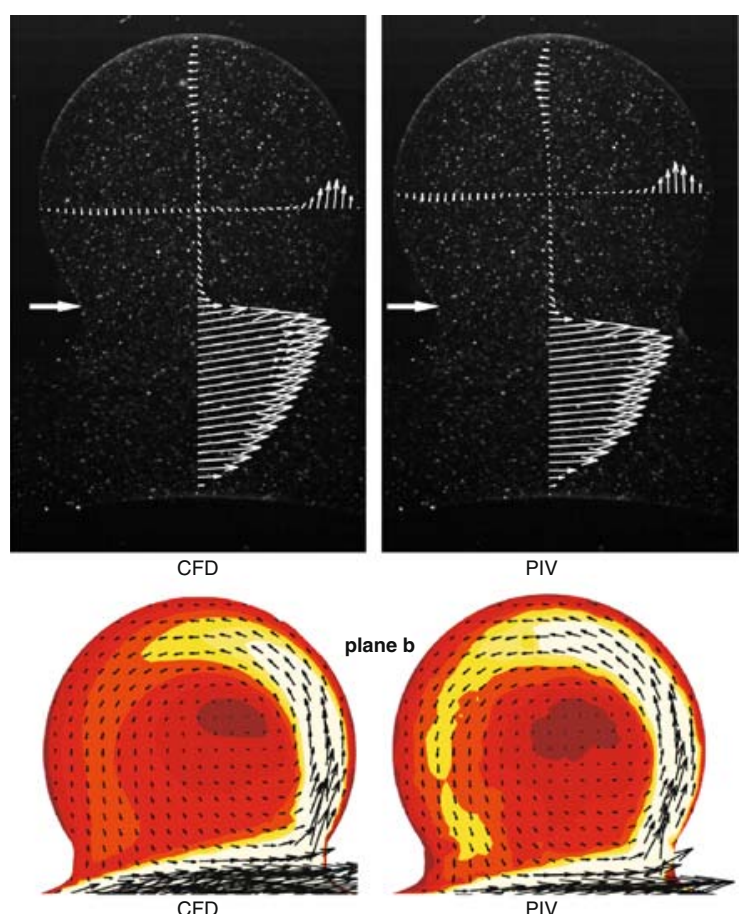

PIV

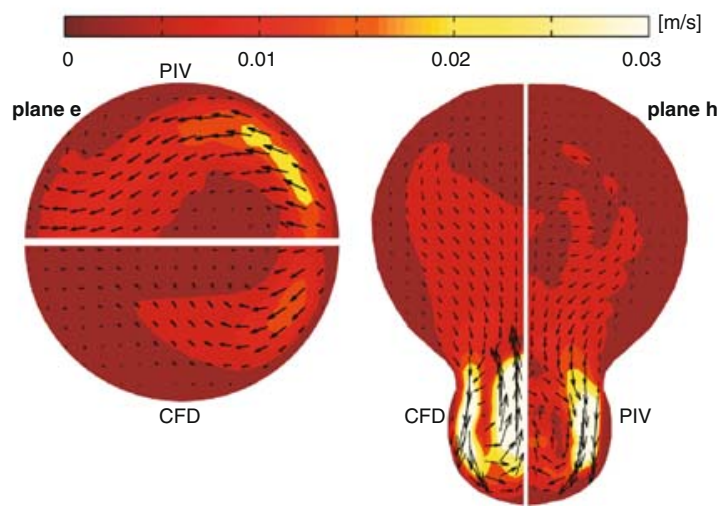

plane, either subsequent frames (plane b) or every tenth frame were correlated. The region of interest is imaged on $570 \times 368$ pixels, and the scaling factor is $2.5 \times 10^{-5} \mathrm{~m}$ per pixel for planes $\mathrm{b}$ and $\mathrm{h}$, whereas it is $2.9 \times 10^{-5} \mathrm{~m}$ per pixel for plane e.

The velocity field was computed using an adaptive correlation method [27], which improves the correlation for larger displacements and velocity gradients. In the first incremental step the size of the interrogation areas was $32 \times 32$ pixels, the result of which is used to pre-shift the interrogation areas in the next incremental step in which the interrogation area size equals $16 \times 16$ pixels. Furthermore, every step uses a 50\% overlap of the interrogation areas to reduce loss-of-pairs.

Various methods are available to identify erroneous vectors which are inevitably present in PIV data even when the experiment is conducted carefully [28]. In general, detecting erroneous vectors based on temporal information is not reliable since PIV has a rather low temporal resolution [29]. However, since the measurements were performed with a high frame rate in a stationary flow without any expected transient flow phenomena, the velocity field could be averaged over the 189 measurements performed. 

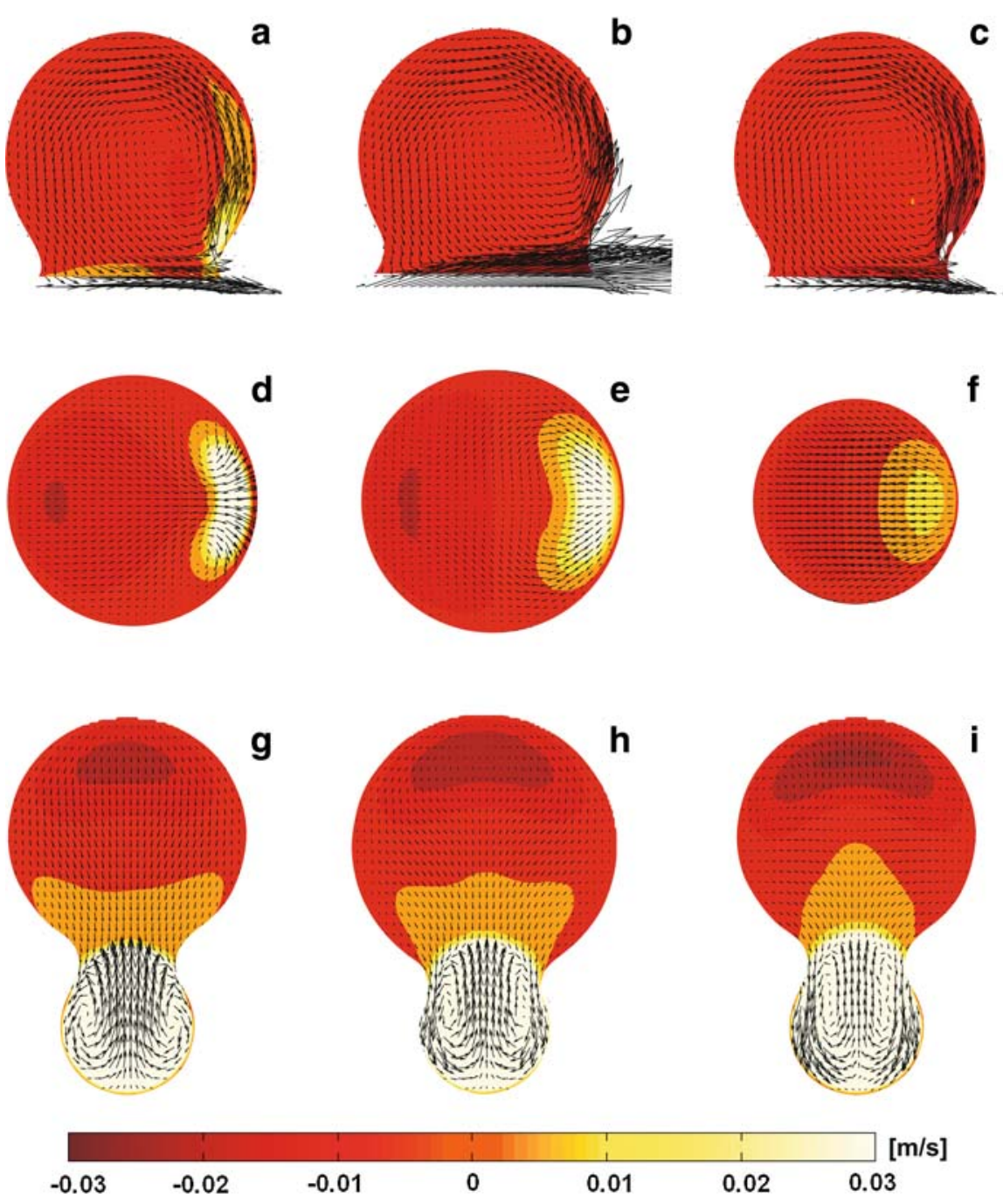

Fig. 5 The velocity field within several cross-sections, of which the numbering is as introduced in Fig. 3. The contours represent the out-of-plane velocities (in $\mathrm{ms}^{-1}$ ). The flow in the parent artery is from left to right in a-f, while the viewing direction in $\mathrm{g}-\mathrm{i}$ is in the direction of the flow

In order to compare the computational and experimental results, the computed velocities were interpolated onto a uniform grid of which the grid size corresponds to the PIV data. Equal Reynolds numbers were used in the experiment and computation. Furthermore, several cross-sections (Fig. 3) were visualized for a more detailed analysis of the 3D velocity field obtained with the CFD model.

\section{Results}

The profiles in Fig. 4 clearly show the slanted profile in the curved parent artery, with higher velocities and velocity gradients near the outer wall. The aneurysmal neck shows a high velocity gradient where the aneurysmal vortex and the flow in the parent artery meet. In order to estimate the quantitative agreement of PIV and CFD, the discrete integrals of the velocity magnitude over the lines shown in Fig. 4 are determined. In the parent artery (below the neck as indicated by the arrow in Fig. 4), this integral is approximately $8 \%$ higher in PIV relative to CFD. In the aneurysm however, the difference is approximately $2 \%$ in both directions. 


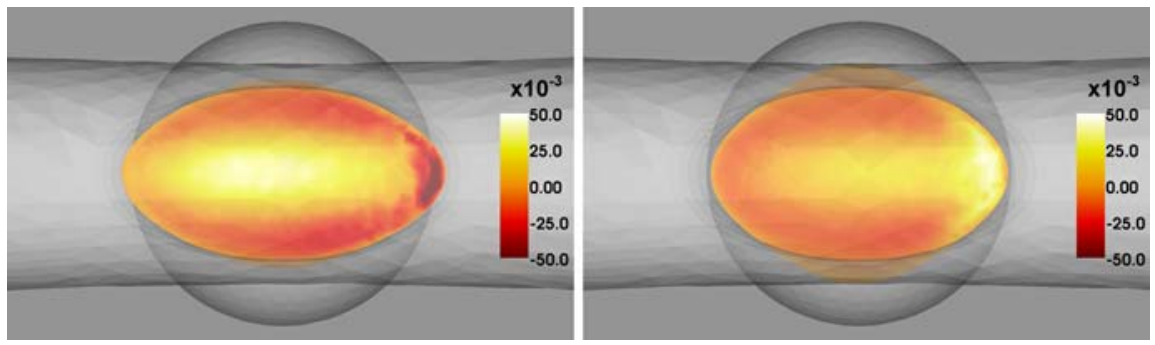

Fig. 6 Bottom view of the out-of-plane velocity (in ms ${ }^{-1}$ ) in the lower part of the neck (left) and $0.3 \mathrm{~mm}$ higher, at the upper part of the neck (right). Since these cross-sections are localized just above and underneath the site where the inflow jet meets the distal lip of the neck, the light area at the distal lip in the top cross-section represents the inflow in the aneurysms while the red area in the lower cross-section represents the fluid that goes straight back into the parent artery and never entered the aneurysm. Inflow occurs mainly in the symmetry plane, whereas outflow occurs along the wall. The flow into the parent artery is from left to right

The contours in Fig. 4 represent the magnitude of the in-plane velocity determined with CFD and PIV. The measured velocities are slightly higher than the computed velocities. Both velocity fields in plane b (Fig. 4) depict a single vortex structure in the aneurysmal sac, with the vortex center located distally to the aneurysm center. Furthermore, the velocities and velocity gradients near the distal wall are much higher compared to those at the proximal wall. Plane h (Fig. 4) reveals secondary flow patterns in the curved parent artery, although the velocity in the center of the parent artery is not captured by the PIV measurements. The flow patterns (e.g. vortex core in the top of the aneurysmal sac) observed in the velocity field obtained with PIV do not appear in the computed velocity field. In plane e (Fig. 4), however, the features show good agreement.

While with PIV only three planes are measured, CFD allows a more detailed analysis of multiple planes in the 3D velocity field. Figure 5 shows the velocity field in the cross-sections defined in Fig. 3, with the contours representing the out-of-plane velocity. The velocity fields in Fig. 5a-c depict a single vortex structure as described above.

Inflow occurs mainly at the distal lip of the neck close to the plane of symmetry (Fig. 5b, d). As the inflow jet meets the distal lip, a portion of the flow is directed into the aneurysm, whereas most fluid follows the flow in the parent artery. This is clearly visible from the out-of-plane velocity in two cross-sections in the aneurysmal neck (Fig. 6), just above and underneath the site where the inflow jet meets the distal lip. At the bottom of the neck the velocity at the distal lip is directed towards the parent artery (red), while the cross-section at the top of the neck shows flow into the aneurysm (white). The inflow jet widens as it spreads over the distal wall, initiating two small vortex cores on each side of the symmetry plane at the distal side of the aneurysm (Fig. 5d, e). In the upper part of the aneurysm the fluid follows the wall (Fig. 5f).

Figure $5 \mathrm{~g}-\mathrm{i}$ depicts a shift of the secondary flow pattern in the parent artery towards the aneurysm as it proceeds along the neck, allowing outflow along the wall (see also Fig. 6). In the proximal cross-section (Fig. 5g) the flow follows the aneurysm wall, while the velocity is directed towards the symmetry plane in the middle of the aneurysm (Fig. 5h). In the lower part of the aneurysm, the velocity is re-diverted towards the wall as it meets the flow in the parent artery. Figure 5i shows the cross-section in close proximity to the vortex center, which depicts a complex flow pattern. In the lower part the flow is directed towards the parent artery, whereas it is directed towards the top in the upper part of the aneurysm. As the fluid meets the top of the aneurysm it spreads along the wall, only to be directed towards the symmetry plane just below of the vortex center. This results in yet another set of small vortex cores on each side of the plane of symmetry.

The contours in Fig. 7 represent the area in which the second largest eigenvalue, $\lambda_{2}$, is smaller than zero. The velocity fields in Fig. 5a-c show a single vortex, whereas the vortex identification depicts a more complex vortex structure (Fig. 7a-c). When considering the velocity fields in other cross-sections, it becomes evident that there are other smaller vortex cores present as described above (Fig. $5 \mathrm{~d}-\mathrm{i}$ ). This clearly shows the complexity of the 3D vortex structure, in which smaller vortices interact with each other. Altogether, the volume containing the vortex core is a doubly curved structure where both curvatures have the same sign, resulting in an indented sphere. 

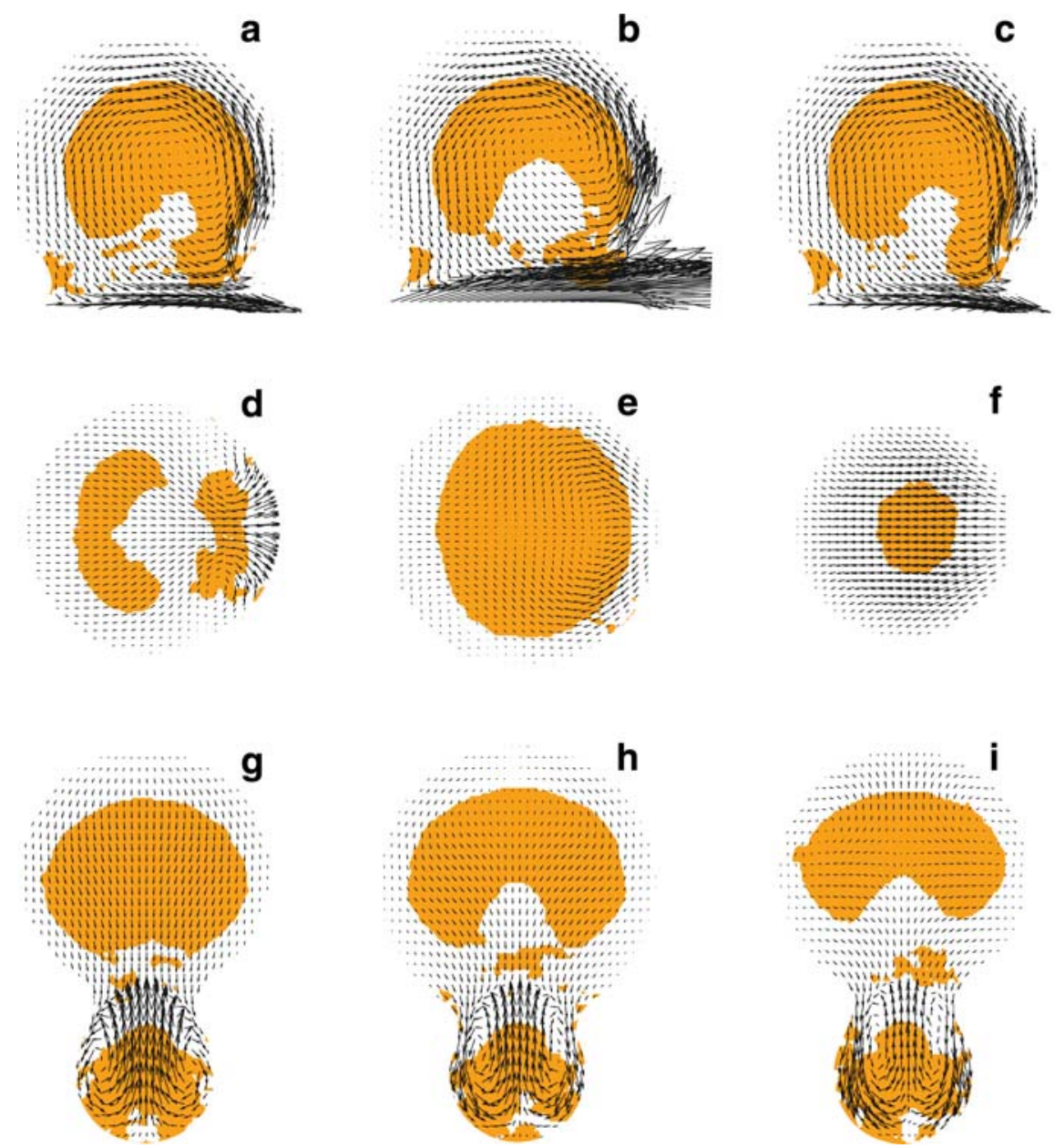

Fig. 7 Velocity vectors and vortex structures in the cross-sections defined in Fig. 3. Detection of the vortex core is achieved using a vortex identification scheme based on the second eigenvalue method developed by Jeong and Hussain [19]

\section{Discussion}

It is well-known that hemodynamical forces are involved in aneurysm growth and rupture. Unfortunately, intraaneurysmal flow can currently not be determined in vivo. CFD is a powerful tool in the analysis of intra-aneurysmal flow patterns, in both idealized and patient-specific geometries. However, several assumptions have to be made in the modeling of this pathology, as in all biological systems. First of all, Newtonian behavior was assumed when modeling the blood flow, which is reasonable for the high flows observed in large arteries. Within the aneurysmal sac however, velocities may become very low which might introduce the need for a more realistic viscosity model. Furthermore, a rigid wall is assumed, which obviously is not the case in vivo [30]. The compliance of the vessel wall might be incorporated in the model, but that would require knowledge of the pressure and mechanical properties of the wall. These properties can not be measured directly in vivo. However, as the resolution of visualization modalities increases, it might become possible to derive them from wall motion and pressure measurements in the future. These pressure measurements would also be relevant when determining appropriate boundary conditions, since the stress-free outflow boundary condition used here will not suffice when wall compliance is taken into account. Altogether, the number of unknowns introduced when the wall compliance is taken into account may cause this more complex model to be less accurate. 
The velocity fields obtained with the CFD model are compared to those measured with PIV in three cross-sections (Fig. 3). In PIV measurements the high out-of-plane velocities are a major complication, especially if the in-plane velocities are relatively low. Hence, the measurements in-plane $\mathrm{b}$ were expected to be most accurate. Indeed, the magnitude of the measured intra-aneurysmal velocities are only $2 \%$ higher than the computed velocities. Since the PIV settings were based on the velocities in aneurysm, a larger difference is observed in the parent artery. However, the slanted profile in the parent artery (Fig.4) is consistent with theoretical considerations of flow in curved tubes [31,32].

In planes e and h the out-of-plane velocities are higher than the in-plane velocities, especially near the distal side of the aneurysm and in the parent artery. Still, PIV captures the secondary flow pattern along the wall of the parent artery, whereas the out-of-plane velocity is too high in the center of the parent artery. The characteristics in the measured velocity field in plane h (Fig. 4) resembles the velocity field in planes i (Fig. 5). For planes e and h, the flow patterns observed $1 \mathrm{~mm}$ next to the plane considered differ significantly, whereas this is not the case for plane b. Hence, when measuring planes e and $\mathrm{h}$, a slight deviation in the positioning of the laser sheet, and averaging of the velocities over the thickness of the laser sheet, influence the resulting velocity field.

As could be expected based on the low-Reynolds-number, no-transitional flow or flow disturbances are observed in the velocity field obtained with PIV. Therefore, the Euler implicit time integration used in the CFD model is able to describe the steady state limit of the flow. Considering the similarities between the characteristics of the measured and computed velocity fields, it seems that the CFD model describes the velocity fields in the aneurysm and its parent artery correctly. Since this CFD method has been used successfully in other applications [33] and the velocity fields obtained with PIV and CFD show the same characteristics, the CFD model will be used for further analysis of the intra-aneurysmal velocity field.

The intra-aneurysmal velocity fields show a single counterclockwise vortex, which is consistent with the findings of Liou et al. [15]. Furthermore, Liou et al. [15] reported that the inflow proceeds around the distal lip of the neck close to the plane of symmetry, which is also observed in the velocity field within the plane of symmetry (Fig. 5b) and cross-sections in the neck (Fig. 6). When considering the velocity field within the planes in Fig. 5a-c, one would expect the vortex identification to result in a circle in Fig. 7a-c. The 3D flow pattern, as can be seen in the other cross-sections, results in a more complex vortex core structure due to the presence of multiple interacting vortices. This clearly shows the need of a more advanced analysis method for intra-aneurysmal flow patterns, such as the identification and quantification of features like vortices and stagnation regions.

Cebral [8] uses one cross-section to characterize the blood flow patterns in the aneurysmal sac. This may lead to a misinterpretation of the data, since a pattern might appear to be a single vortex in one plane and more complex in the other. This can be observed even in the simplified geometry used here. In pulsatile flow conditions, the location of the vortex cores will shift throughout a cardiac cycle. The smaller vortices observed in Fig. 5d, e and i, if present in pulsatile flow, might have an influence on the oscillating wall shear stress. Therefore, these small vortices may be important in the relation of rupture risk and flow patterns. However, since the major drawback of the vortex identification method used here [19] is the failure to discriminate between different vortex cores that are in close proximity of each other, multiple cores may arise as one vortex structure. Another vortex identification method, e.g. the predictor-corrector method proposed by Banks and Singer [34], or combination of vortex identification methods, might be more appropriate for this application. The relationship between the vortex structure and rupture risk remains to be established. Moreover, other features, such as stagnation regions, may be relevant as well. The methods to be used should be optimized by the analysis of intra-aneurysmal flow patterns in different idealized geometries, after which patient-specific geometries should be examined in order to identify rupture risk estimators.

Open Access This article is distributed under the terms of the Creative Commons Attribution Noncommercial License which permits any noncommercial use, distribution, and reproduction in any medium, provided the original author(s) and source are credited. 


\section{References}

1. Mizoi K, Yoshimoto T, Nagamine Y et al (1995) How to treat incidental cerebral aneurysms: a review of 139 consecutive cases. Surg Neurol 44:114-121

2. Krex D, Schackert HK, Schackert G et al (2001) Genesis of cerebral aneurysms—an update. Acta Neurochir 143:429-449

3. Mitchell P, Birchall D, Mendelow D (2006) Blood pressure, fatigue, and the pathogenisis of aneurysmal subarachnoid hemorrhage. Surg Neurol 66:574-580

4. Juvela S, Poussa K, Porras M (2001) Factors affecting formation and growth of intracranial aneurysms: a long term follow up study. Stroke 32:485-491

5. van Gijn J, Rinkel GJE (2001) Subarachnoid haemorrhage: diagnosis, causes and management. Brain 124:249-278

6. Cebral JR, Castro MA, Burgess JE et al (2005) Characterization of cerebral aneurysms for assessing risk of rupture by using patient-specific computational hemodynamics models. AJNR Am J Neuroradiol 26:2550-2559

7. Castro MA, Putman CM, Cebral JR (2006) Computational fluid dynamics modeling of intracranial aneurysms: effects of parent artery segmentation on intra-aneurysmal hemodynamics. AJNR Am J Neuroradiol 27:1703-1709

8. Cebral JR, Castro MA, Appanaboyina S et al (2005) Efficient pipeline for image-based patient-specific analysis of cerebral aneurysm hemodynamics: technique and sensitivity. IEEE Trans Med Imaging 24(4):457-467

9. Malek A, Izumo S (1995) Control of endothelial cell gene expression by flow. J Biomech 28(12):1515-1528

10. Wentzel J, Gijsen F, Stergiopulos N et al (2003) Shear stress, vascular remodeling and neointimal formation. J Biomech 36:681-688

11. Friedman MH, Fry DL (1993) Arterial permeability dynamics and vascular disease. Atherosclerosis 104:189-194

12. Beck J, Rohde S, Berkefeld J et al (2006) Size and location of ruptured and unruptured intracranial aneurysms measured by 3-dimensional rotational angiography. Surg Neurol 65:18-27

13. Dickley P, Kailasnath P (2002) The diameter-cube hypothesis: a new biophysical model of aneurysm rupture. Surg Neurol 58:166180

14. Ohashi Y, Horikoshi T, Sugita M et al (2004) Size of cerebral aneurysms and related factors in patients with subarachnoid hemorrhage. Surg Neurol 61:239-247

15. Liou TM, Liou SN (1999) A review on in vitro studies of hemodynamic characteristics in terminal and lateral aneurysm models. Proc Natl Sci Counc ROC (B) 23:133-148

16. Steinman DA (2002) Image-based computational fluid dynamics modeling in realistic arterial geometries. Ann Biomed Eng 30:483497

17. Chatziprodromou I, Tricoli A, Poulikakos D et al (2007) Heamodynamics and wall remodelling of a growing cerebral aneurysm: a computational model. J Biomech 40:412-426

18. Cebral JR, Pergolizzi RS, Putman CM (2007) Computational fluid dynamics modeling of intracranial aneurysms: qualitative comparison with cerebral angiography. Acad Radiol 14(7):804-813

19. Jeong J, Hussain F (1995) On the identification of a vortex. J Fluid Mech 285:69-94

20. Castro MA, Putman CM, Cebral JR (2006) Patient-specific computational modeling of cerebral aneurysms with multiple avenues of flow from 3d rotational angiography images. Acad Radiol 13(7):811-821

21. Castro MA, Putman CM, Cebral JR (2006) Patient-specific computational fluid dynamics modeling of anterior communicating artery aneurysms: a study of the sensitivity of intra-aneurysmal flow patterns to flow conditions in the carotid arteries. AJNR Am J Neuroradiol 27:2061-2068

22. Parlea L, Fahrig R, Holdsworth DH et al (1999) An analysis of the geometry of saccular intracranial aneurysms. AJNR Am J Neuroradiol 20:1079-1089

23. Liou TM, Liou SN (2004) Pulsatile flows in a lateral aneurysm anchored on a stented and curved parent vessel. Exp Mech 44:253160

24. Narracott A, Lawford P, Liu H et al (2003) Development of a model for the investigation of blood clotting in cerebral aneurysms following coiling. In: Proc Int Congr Comput Bioeng, Zaragosa, September 24-26, 2003

25. Rudin S, Wang Z, Kyprianou I et al (2003) Imaging stent-induced flow modification in cerebro-vascular aneurysms with X-ray micro-angiography. In: IEEE Int Symp Biomed Imaging, Washington DC, July 7-10, 2002

26. Segal A (2006) Sepran user manual and programmers guide. Ingenieursbureau Sepra, Leidschendam

27. van der Graaf G (2007) Gpiv, open source software for particle image velocimetry. http://gpiv.sourceforge.net/. Accessed 15 Dec 2008

28. Westerweel J (1994) Efficient detection of spurious vectors in particle image velocimetry data. Exp Fluids 16(3-4):236-247

29. Shinneeb AM, Bugg JD, Balachandar R (2004) Variable threshold outlier identification in piv data. Meas Sci Technol 15:1722-1732

30. Meyer FJH, Riederer S (1993) Pulsatile increase in aneurysm size determined by cine phase-contrast mr angiography. J Neurosurg 78:879-883

31. Dean WR (1927) Note on the motion of fluid in a curved pipe. Philos Mag 4(20):208-222

32. Dean WR (1928) The stream-line motion of fluid in a curved pipe. Philos Mag 5(30):673-695

33. van de Vosse FN, de Hart J, van Oijen CHGA et al (2003) Finite-element-based computational methods for cardiovascular fluidstructure interaction. J Eng Math 47:335-368

34. Banks DC, Singer BA (1995) A predictor-corrector technique for visualizing unsteady flow. IEEE Trans Vis Comput Graph 1(2):151-163 